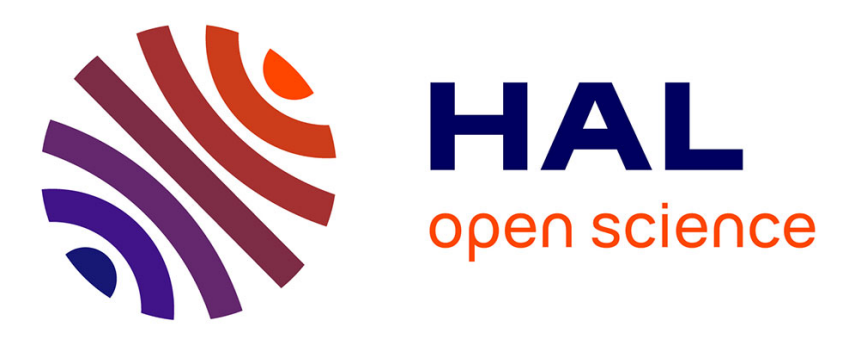

\title{
Possibilistic vs. Relational Semantics for Logics of Incomplete Information
}

Mohua Banerjee, Didier Dubois, Lluis Godo

\section{To cite this version:}

Mohua Banerjee, Didier Dubois, Lluis Godo. Possibilistic vs. Relational Semantics for Logics of Incomplete Information. 15th International Conference, Information Processing and Management of Uncertainty in Knowledge-based Systems - IPMU 2014, Jul 2014, Montpellier, France. pp. 335-344. hal-01147237

\section{HAL Id: hal-01147237 https://hal.science/hal-01147237}

Submitted on 30 Apr 2015

HAL is a multi-disciplinary open access archive for the deposit and dissemination of scientific research documents, whether they are published or not. The documents may come from teaching and research institutions in France or abroad, or from public or private research centers.
L'archive ouverte pluridisciplinaire HAL, est destinée au dépôt et à la diffusion de documents scientifiques de niveau recherche, publiés ou non, émanant des établissements d'enseignement et de recherche français ou étrangers, des laboratoires publics ou privés. 


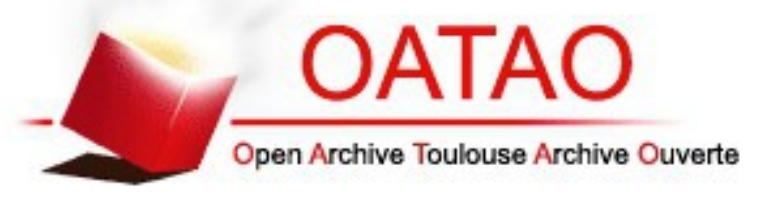

\section{Open Archive TOULOUSE Archive Ouverte (OATAO)}

OATAO is an open access repository that collects the work of Toulouse researchers and makes it freely available over the web where possible.

This is an author-deposited version published in : http://oatao.univ-toulouse.fr/ Eprints ID : 13204

To link to this article : DOI :10.1007/978-3-319-08795-5_35

URL : http://dx.doi.org/10.1007/978-3-319-08795-5 35

To cite this version : Banerjee, Mohua and Dubois, Didier and Godo, Lluis Possibilistic vs. Relational Semantics for Logics of Incomplete Information. (2014) In: 15th International Conference, Information Processing and Management of Uncertainty in Knowledge-based Systems - IPMU 2014, 15 July 2014 - 19 July 2014 (Montpellier, France).

Any correspondance concerning this service should be sent to the repository administrator: staff-oatao@,listes-diff.inp-toulouse.fr 


\title{
Possibilistic vs. Relational Semantics for Logics of Incomplete Information
}

\author{
Mohua Banerjee $^{1}$, Didier Dubois ${ }^{2}$, and Lluis Godo ${ }^{3}$ \\ 1 Dept. of Mathematics and Statistics, Indian Institute of Technology, Kanpur, India \\ 2 IRIT-CNRS, Université Paul Sabatier, Toulouse, France \\ 3 IIIA-CSIC, Bellaterra, Spain
}

\begin{abstract}
This paper proposes an extension of the MEL logic to a language containing modal formulae of depth 0 or 1 only. MEL is a logic of incomplete information where an agent can express both beliefs and explicitly ignored facts, that only uses modal formulae of depth 1, and no objective ones. The extended logic, called $\mathrm{MEL}^{+}$has the same axioms as, and is in some sense equivalent to, S5 with a restricted language, but with the same expressive power. The semantics is not based on Kripke models with equivalence relations, but on pairs made of an interpretation (representing the real state of facts) and a non-empty set of possible interpretations (representing an epistemic state). Soundness and completeness are established. We provide a rationale for using our approach when an agent reasons about what is known of the epistemic state of another agent and compares it with what is known about the real world. Our approach can be viewed as an alternative to the basic epistemic logic not concerned with introspection. We discuss the difference with S5 used as a logic for rough sets, and the similarity with some previous non-monotonic logics of knowledge is highlighted.
\end{abstract}

Keywords: Modal logic, possibility theory, epistemic logic, rough sets.

\section{Introduction}

In the recent past $[1,2]$, some efforts have been made to relate possibility theory and modal logic under the simplest possible syntactic and semantic framework. The resulting language of this logic called MEL is a fragment of KD where no objective formula (not preceded by a modality) appears, and where modalities cannot be nested. Models of MEL are simply non-empty subsets of interpretations of some standard propositional language. They represent the possible epistemic states of some agent. Then the necessity modality represents belief, and an agent believes a proposition if and only if the latter is true in all propositional interpretations compatible with the agent's epistemic state. This logic has axioms $\mathrm{K}$ and $\mathrm{D}$, and another axiom saying that the agent believes all tautologies. Each epistemic state can be interpreted as a Boolean possibility distribution, and it can be shown that the necessity modality is a Boolean necessity measure. This attempt to relate possibility theory and modal logic contrasts with other 
previous connections between these notions, involving more elaborate constructions $[8,9,3,10]$.

The MEL logic has a language which is a fragment of the language of the logic S5 often used as an epistemic logic. Its semantics does not use accessibility relations explicitly, and is much simpler. Moreover one cannot express the introspective axioms 4 and 5 in MEL as the nesting of modalities is not possible in its language. Nevertheless, two issues are worth investigating in connection with MEL:

- Is it possible to extend the language of MEL to objective non-modal formulae, while preserving the same style of semantics as MEL ?

- Given that standard epistemic logics such as S5 rely on accessibility (equivalence) relations, what is the connection between these two semantics?

The aim of this paper is to provide an answer to both questions. In the next section, a brief presentation of the logic MEL is recalled. Then an extension of the MEL language to objective formulae and the corresponding extension of the MEL semantics is proposed. Finally we discuss the usual Kripke style semantics of S5 and compare them to our semantics, which we consider more natural than the relational semantics based on indiscernible possible worlds according to an equivalence relation. We claim that the latter semantics is more fit to rough sets and can account for the idea of forgetting [11]. Moreover, we show that our approach comes closer to some knowledge logics proposed in the early 1990's, in the area of non-monotonic reasoning.

\section{MEL, A Simple Epistemic Logic}

The usual truth values true (1) and false (0) assigned to propositions are of ontological nature (which means that they are part of the definition of what we call proposition), whereas assigning to a proposition a value whose meaning is expressed by the word unknown sounds like having an epistemic nature: it reveals a knowledge state according to which the truth value of a proposition (in the usual Boolean sense) in a given situation is out of reach (for instance one cannot compute it, either by lack of computing power, or due to a sheer lack of information). It corresponds to an epistemic state for an agent that can neither assert the truth of a Boolean proposition nor its falsity.

Admitting that the concept of "unknown" refers to a knowledge state rather than to an ontic truth value, we may keep the logic Boolean and add to its syntax the capability of stating that we ignore the truth value ( 1 or 0 ) of propositions. The natural framework to syntactically encode statements about knowledge states of propositional logic (PL) statements is modal logic, and in particular, the logic KD. Nevertheless, if one only wants to reason about e.g. the beliefs of another agent, a very limited fragment of this language is needed. The logic MEL $[1,2]$ was defined for that purpose.

Let us consider $\mathcal{L}$ to be a standard propositional language built up from a finite set of propositional variables $\mathcal{V}=\left\{p_{1}, \ldots, p_{k}\right\}$ along with the Boolean 
connectives of conjunction and negation $\neg$. As usual, a disjunction $\varphi \vee \psi$ stands for $\neg(\neg \varphi \wedge \neg \psi)$ and an implication $\varphi \rightarrow \psi$ stands for $\neg \varphi \vee \psi$. Further we use $\top$ to denote $\varphi \vee \neg \varphi$, and $\perp$ to denote $\neg \top$. Let us consider another propositional language $\mathcal{L}_{\square}$ whose set of propositional variables is of the form $\mathcal{V}_{\square}=\{\square \varphi \mid$ $\varphi \in \mathcal{L}\}$ to which the classical connectives can be applied. It is endowed with a modality operator expressing certainty, that encapsulates formulae in $\mathcal{L}$. In other words $\mathcal{L}_{\square}=\{\square \alpha: \alpha \in \mathcal{L}\}|\neg \Phi| \Phi \wedge \Psi$.

MEL is a logic on the language $\mathcal{L}_{\square}$ and with the following semantics. Let $\Omega$ be the set of classical interpretations for the propositional language $\mathcal{L}$, i.e. $\Omega$ consists of the set of mappings $w: \mathcal{L} \rightarrow\{0,1\}$ conforming to the rules of classical propositional logic. For a propositional formula $\varphi \in \mathcal{L}$ we will denote by $\operatorname{Mod}(\varphi)$ the set of $w \in \Omega$ such that $w(\varphi)=1$. Models (or interpretations) for MEL correspond to epistemic states, which are simply subsets $\emptyset \neq E \subseteq \Omega$. The truth-evaluation rules of formulas of $\mathcal{L}_{\square}$ in a given epistemic model $E$ is defined as follows:

$-E \models \square \varphi$ if $E \subseteq \operatorname{Mod}(\varphi)$

$-E \models \neg \Phi$ if $E \not \models \Phi$

- $E \models \Phi \wedge \Psi \quad$ if $\quad E \models \Phi$ and $E \models \Psi$

Note that contrary to what is usual in modal logic, we do not evaluate modal formulas on particular interpretations of langage $\mathcal{L}$ because modal formulas in MEL do not refer to the actual world.

The notion of logical consequence is defined as usual $\Gamma \models \Phi$ if, for every epistemic model $E, E \models \Phi$ whenever $E \models \Psi$ for all $\Psi \in \Gamma$.

MEL can be axiomatized in a rather simple way (see [2]). The following are a possible set of axioms for MEL in the language of $\mathcal{L}_{\square}$ :

(PL) Axioms of PL for $\mathcal{L}_{\square}$-formulas

(K) $\square(\varphi \rightarrow \psi) \rightarrow(\square \varphi \rightarrow \square \psi)$

(D) $\square \varphi \rightarrow \diamond \varphi$

(Nec) $\square \varphi$, for each $\varphi \in \mathcal{L}$ that is a PL tautology, i.e. if $\operatorname{Mod}(\varphi)=\Omega$.

The only inference rule is modus ponens. The corresponding notion of proof, denoted by $\vdash$, is defined as usual from the above set of axioms and modus ponens.

This set of axioms provides a sound and complete axiomatization of MEL, that is, it holds that, for any set of MEL formulas $\Gamma \cup\{\varphi\}, \Gamma \models \varphi$ iff $\Gamma \vdash \varphi$. This is not surprizing: MEL is just a standard propositional logic with additional axioms, whose propositional variables are the formulas of another propositional logic, and whose interpretations are subsets of interpretations of the latter.

Notice that MEL also actually captures the $\mathcal{L}_{\square}$-fragment of the normal modal logics KD, hence of other logics, such as the well-known logics KD45 and S5, also commonly referred to as the logics of belief and knowledge, respectively. However, they are obtained from KD with axioms (called 4, 5, T) that cannot be expressed in the MEL language $\mathcal{L}_{\square}$. 


\section{Extending MEL to Reason about The Actual World and Someone's Beliefs}

In this section we extend the language of MEL to allow dealing with not only subjective formulas that express an agent's beliefs, but also objective formulas (i.e. non-modal formulas) that express propositions that hold true in the actual world (whatever it might be). The extended language will be denoted by $\mathcal{L}_{\square}^{+}$, and it thus contains both propositional and modal formulas. It exactly corresponds to the non-nested fragment of the language of usual modal logic.

\subsection{Language, Axioms and Semantics}

More precisely, the language $\mathcal{L}_{\square}^{+}$of $\mathrm{MEL}^{+}$extends $\mathcal{L}_{\square}$ and is defined by the following formation rules:

- If $\varphi \in \mathcal{L}$ then $\varphi, \square \varphi \in \mathcal{L}_{\square}^{+}$

- If $\Phi, \Psi \in \mathcal{L}_{\square}^{+}$then $\neg \Phi, \Phi \wedge \Psi \in \mathcal{L}_{\square}^{+}$

$\diamond \varphi$ is defined as an abbreviation of $\neg \square \neg \varphi$. Note that $\mathcal{L} \subseteq \mathcal{L}_{\square}^{+}$and that in $\mathcal{L}_{\square}^{+}$ there are no formulas with nested modalities.

Semantics for $\mathrm{MEL}^{+}$are given now by "pointed" MEL epistemic models, i.e. by structures $(w, E)$, where $w \in \Omega$ and $\emptyset \neq E \subseteq \Omega$. The truth-evaluation rules of formulas of $\mathcal{L}_{\square}^{+}$in a given structure $(w, E)$ is defined as follows:

- $(w, E) \models \varphi$ if $w(\varphi)=1$, in case $\varphi \in \mathcal{L}$

- $(w, E) \models \square \varphi$ if $E \subseteq \operatorname{Mod}(\varphi)$

- usual rules for $\neg$ and $\wedge$

Logical consequence, as usual: $\Gamma \models \Phi$ if, for every structure $(w, E),(w, E) \models \Phi$ whenever $(w, E) \models \Psi$ for all $\Psi \in \Gamma$.

The following are the axioms for $\mathrm{MEL}^{+}$in the language of $\mathcal{L}_{\square}^{+}$:

(PL) Axioms of propositional logic

(K) $\square(\varphi \rightarrow \psi) \rightarrow(\square \varphi \rightarrow \square \psi)$

(D) $\square \varphi \rightarrow \diamond \varphi$

(Nec) $\square \varphi$, for each $\varphi \in \mathcal{L}$ that is a PL tautology, i.e. if $\operatorname{Mod}(\varphi)=\Omega$.

The only inference rule is modus ponens. ${ }^{1}$

\subsection{Completeness}

In what follows, we will denote by $\vdash_{P L}$ the notion of proof of classical propositional language on the language $\mathcal{L}_{\square}^{+}$taking all $\square$-formulas as new propositional variables. We will further let $\Gamma \cup\{\Phi\}$ be a set of $\mathcal{L}_{\square}^{+}$-formulas. We need first to recall the following lemma [2].

\footnotetext{
${ }^{1}$ An equivalent presentation could be to replace (Nec) by the usual Necessitation rule in modal logics, but restricted to tautologies of propositional logic: if $\varphi \in \mathcal{L}$ is a theorem, derive $\square \varphi$.
} 
Lemma 1. $\Gamma \vdash \Phi$ iff

$\Gamma \cup\left\{\square \varphi \mid \vdash_{P L} \varphi\right\} \cup\{$ instances of axioms $(K),(D)$ and $(N e c)\} \vdash_{P L} \Phi$

Theorem 1. $\Gamma \vdash \Phi$ iff $\Gamma \models \Phi$.

Proof. From left to right is easy, as usual. For the converse direction, assume $\Gamma \forall \Phi$. By the preceding lemma and the completeness of PL, there exists a propositional evaluation $v$ on the whole language $\mathcal{L}_{\square}^{+}$(taking $\square$-formulas as genuine propositional variables) such that $v(\Psi)=1$ for all $\Psi \in \Gamma \cup\left\{\square \varphi \mid \vdash_{P L}\right.$ $\varphi\} \cup\{$ instances of axioms $(\mathrm{K})$ and $(\mathrm{D})\}$ but $v(\Phi)=0$. We have to build a structure $(w, E)$ that it is a model of $\Gamma$ but not of $\Phi$. So, we take $(w, E)$ as follows:

- $w$ is defined as the restriction of $v$ to $\mathcal{L}$, i.e. $w(\varphi)=v(\varphi)$ for all $\varphi \in \mathcal{L}$.

$-E=\bigcap\{\operatorname{Mod}(\varphi) \mid v(\square \varphi)=1\}$

Note that, since by assumption $v(\diamond \top)=1, E \neq \emptyset$. Then the last step is to show that, for every $\Psi \in \mathcal{L}_{\square}^{+}, v(\Psi)=1$ iff $(w, E) \models \Psi$. We prove this by induction. The case $\Psi$ being a non-modal formula from $\mathcal{L}$ is clear, since in that case $w(\Psi)=v(\Psi)$. The interesting case is when $\Psi=\square \psi$. Then we have:

(i) If $v(\square \psi)=1$ then, by definition of $E, E \subseteq \operatorname{Mod}(\psi)$, and hence $(w, E) \models \square \psi$.

(ii) Conversely, if $E \subseteq \operatorname{Mod}(\psi)$, then there must exist $\gamma$ such that $v(\square \gamma)=1$ and $\operatorname{Mod}(\gamma) \subseteq \operatorname{Mod}(\psi)$. Hence this means that $\gamma \rightarrow \psi$ is a PL theorem, and hence we have first, by the necessitation axiom, that $v(\square(\gamma \rightarrow \psi))=1$, and thus $v(\square \gamma) \leq v(\square \psi)$ holds as well by axiom $(\mathrm{K})$, and therefore $v(\square \psi)=1$ holds as well.

As a consequence, we have that $(w, E) \models \Psi$ for all $\Psi \in \Gamma$ and $(w, E) \not \models \Phi$.

Remark 1. Notice that if the notion of logical consequence $\models$ is reduced to considering only structures $(w, E)$ such that $w \in E$, then one should add the following well-known axiom (T): $\square \varphi \rightarrow \varphi$ to keep completeness.

\section{Relating MEL and MEL ${ }^{+}$to KD45 and S5}

Recall the normal modal systems KD, KD4, KD45 and S5 (see e.g. [4] for details).

Proposition 1. Let $\varphi$ a formula from $\mathcal{L}_{\square}$. Then $M E L \vdash \varphi$ iff $K D \vdash \varphi$.

Proof. Assume $K D \forall \varphi$, then there is a serial Kripke model $(W, e, R)$ and $w \in W$ such that $e(w, \varphi)=0$. Since $\varphi$ does not contain nested modal operators, $e(w, \varphi)$ only depends on the truth-evaluations of subformulas of $\varphi$ at all the worlds from $R(w)=\left\{w^{\prime} \mid w R w^{\prime}\right\}$, which is non-empty since $R$ is serial. We can assume $R(w) \subseteq \Omega$. Define, for each $w^{\prime} \in R(w)$, the propositional evaluation $v_{w^{\prime}}():.=$ $e\left(w^{\prime},.\right)$, and the epistemic model $E_{w}:=\left\{v_{w^{\prime}}: w^{\prime} \in R(w)\right\}$. Then $E_{w} \models \varphi$, if and only if $e(w, \varphi)=1$. Hence MEL $\forall \varphi$.

Conversely, assume MEL $\forall \varphi$, then there is an epistemic model $E$ such that $E \not \forall \varphi$. Now, consider the Kripke model $M=(E, e, R)$ where $R=E \times E$ with $e(v, \cdot)=v(\cdot)$ for every $v \in E$. $M$ is clearly a serial model. The fact that $E \not \forall \varphi$ implies that $e(w, \varphi)=0$ for some $w \in E$, and hence $M \forall \varphi$, and by completeness, $K D \forall \varphi$. 
Corollary 1. Let $\varphi$ a formula from $\mathcal{L}_{\square}$. Then $M E L \vdash \varphi$ iff $L \vdash \varphi$ for $L \in$ $\{K D 4, K D 45, S 5\}$.

Now let us consider the case of $\mathrm{MEL}^{+}$. Then we have even stronger relationships to KD45 and S5.

Proposition 2. Let $\varphi$ a formula from $\mathcal{L}_{\square}^{+}$. Then $M E L^{+} \vdash \varphi$ iff $K D \vdash \varphi$.

Proof. The proof is very similar to that of Proposition 1.

Corollary 2. Let $\varphi$ a formula from $\mathcal{L}_{\square}^{+}$. Then $M E L^{+} \vdash \varphi$ iff $L \vdash \varphi$ for $L \in$ $\{K D 4, K D 45\}$.

Note that this corollary does not hold for $\mathrm{L}=\mathrm{S} 5$, indeed, $\square \varphi \rightarrow \varphi$ is an axiom of S5 that is not provable in $\mathrm{MEL}^{+}$.

Let us call $\mathrm{MEL}^{++}$the extension of $\mathrm{MEL}^{+}$with the axiom $(\mathrm{T}): \square \varphi \rightarrow \varphi$. Then notice that an easy adaptation of the proof of completeness theorem for $\mathrm{MEL}^{+}$proves that $\mathrm{MEL}^{++}$is complete with respect to the class of reflexive pointed epistemic models $(w, E)$ where $w \in E$.

Proposition 3. Let $\varphi$ a formula from $\mathcal{L}_{\square}^{+}$. Then, $M E L^{++} \vdash \varphi$ iff $S 5 \vdash \varphi$.

The proof easily follows from that of Proposition 2 by taking into account that one has to deal with reflexive epistemic models.

Moreover, by recalling the well-known result that any formula of KD45 and S5 is logically equivalent to another formula without nested modalities, we can formulate the following stronger relationships.

Proposition 4. The following conditions hold true:

- For any arbitrary modal formula $\varphi$, there is a formula $\varphi^{\prime} \in \mathcal{L}_{\square}^{+}$such that $K D 45 \vdash \varphi$ iff $M E L^{+} \vdash \varphi^{\prime}$.

- For any arbitrary modal formula $\varphi$, there is a formula $\varphi^{\prime} \in \mathcal{L}_{\square}^{+}$such that $S 5 \vdash \varphi$ iff $M E L^{++} \vdash \varphi^{\prime}$.

Remark 2. Recently, Petruszczak [16] indicated that simplified Kripke frames could indeed be used for the semantics of systems K45, KB5 and KD45, using subsets of propositional valuations in place of relations, as we proposed. He proves it by constructing specific accessibility relations equivalent to such subsets, as in [1] for MEL, while the completeness proof in [2] and here is direct.

\section{$5 \mathrm{MEL}^{+}$vs. Other Logics of Incomplete Information}

The language MEL is supposed to encode the following situation [1]. There are two agents, one of which, say $\mathcal{A}$, reasons about some beliefs possessed by another agent $\mathcal{B}$, the former is aware of, on the basis of the testimony of the latter. Namely, $\mathcal{A}$ partially knows what the other agent believes. A belief base in MEL typically contains the testimony of agent $\mathcal{B}$, namely propositions agent 
$\mathcal{B}$ believes $(\square \alpha)$, some that he explicitly does not know $(\diamond \alpha \wedge \diamond \neg \alpha)$, and finally some other propositions that agent $\mathcal{A}$ is aware the agent $\mathcal{B}$ knows the truthvalue of, without guessing which $(\square \alpha \vee \square \neg \alpha)$. The logic MEL enables agent $\mathcal{A}$ to infer more beliefs agent $\mathcal{B}$ possesses but did not reveal. Such a (meta-)belief base for agent $\mathcal{A}$ is equivalent to a set of possible epistemic states for agent $\mathcal{B}$. In $\mathrm{MEL}^{+}$, agent $\mathcal{A}$ is allowed to add what is known about the real world in the form of standard propositions. So $\alpha \wedge \square \neg \alpha$ means that agent $\mathcal{A}$ considers $\alpha$ is true, while he knows that agent $\mathcal{B}$ believes it is false. Under this set-up, a MEL ${ }^{+}$ model $(w, E)$ is interpreted as the fact that $\mathcal{A}$ envisages the real world to be $w$ and the epistemic state of $\mathcal{B}$ to be $E$. If $\mathcal{A}$ considers that $\mathcal{B}$ 's beliefs are always correct, the former can assume axiom $\mathrm{T}$ is valid, thus he reasons in $\mathrm{MEL}^{++}$to strengthen his own knowledge of the real world. Alternatively, $\mathcal{A}$ may mistrust $\mathcal{B}$ and may wish to take advantage of knowing wrong beliefs of $\mathcal{A}$.

\subsection{Epistemic Logic and Accessibility Relations: A Critique}

In contrast, usual semantics of S5 [7] consider the epistemic state of an agent is modelled by an equivalence relation $R$ on a set of possible worlds $W{ }^{2}$ The statement $w R w^{\prime}$ reads "world $w^{\prime}$ is accessible from $w$ ". The world $w^{\prime}$ is said to be an epistemic or doxastic alternative to world $w$ for the agent, depending on whether knowledge or belief is the considered attitude. There are various attempts to make sense of this relation, such that the agent cannot distinguish $w$ from $w^{\prime}$, or $w^{\prime}$ is a possible state of affairs from the point of view of what the agent knows in $w$, etc. The underlying idea seems to be that "the set of worlds considered possible by an agent depends on his or her informational resources at that instant" (Stanford Encyc. Philos.).

However this view, which seems to be shared by many scholars, is not so easy to grasp. Interpreting "accessible worlds" as worlds compatible with the agent epistemic state, we can assume that the epistemic state of the agent depends on his or her informational resources at that instant (in MEL this possibility is not considered). But it is not clear that the agent is aware of his own informational resources to the point of articulating them in the same language as the one he uses to speak about the current states of affairs. If $w$ stands for an objective state of facts, it may not include the particulars of the agent. The epistemic state of the agent depends on many hidden internal features of the agent but his current observations about the actual world $w$ refer to something external, the agent is focused on. It is not clear that the vocabulary used to describe the actual world $w$ is rich enough to also account for the inner state (of health or informational resources) of the agent that holds some beliefs about $w$. For instance suppose the agent has incomplete information on the outcome of coin flipping round after the toss: this epistemic state will generally not depend on the outcome of the toss (like "if the result is heads then he knows it, otherwise he does not know").

\footnotetext{
${ }^{2}$ See [6] for an alternative semantics that makes the internal structure of possible worlds more explicit, encoding both objective facts and agents' mental states in a possible world. We thank one reviewer for pointing this out to us.
} 
In a nutshell, while an equivalence class of $R$ represents context-dependent knowledge of the agent, it is not clear that this contextual dependence is part of the agent's knowledge about himself, let alone about another agent. So in the epistemic logic approach the accessibility relation seems to be a circular notion, where possible worlds seem to include the description of the agent mental circumstances as well as the description of his epistemic state regarding the problem he considers. This view may make sense when introspection is the main issue (the agent being partially unaware of his own knowledge), but it seems to be at odds with the problem of an agent reflecting about other agents knowledge, as in the set-up for MEL and $\mathrm{MEL}^{+}$. As the set-up for MEL is not introspective, this relational semantics looks like a questionable artifact for this logic, where we assume agents are aware of their own knowledge and lack of knowledge. Note that if $R=W \times W$ one can only distinguish between tautologies (i.e. $\square \phi$ where $\phi$ is a PL tautology), contradictions and contingent modal propositions.

One may extend the MEL set-up by considering a separate set of possible mental dispositions $S$ corresponding to "informational resources" (due to specific situations or circumstances) an agent can access at a particular moment. On the other hand, $W$ encodes the question the agent is concerned with at that moment; it pertains to the outside world, so $S \cap W=\emptyset$ The accessibility relation $R$ is relating $S$ to $W$, namely $E=R(s) \subseteq W$ is the epistemic state of the agent when his mental disposition is $s$. Note that, under this view, there is no point of $R$ being an equivalence relation. But this extension assumes that the set $S$ of mental dispositions of the agent is known and observable by another agent.

\subsection{Comparison to Logics of Rough Sets}

The semantics of S5 in terms of equivalence relations [4] makes it in fact the natural logical setting for rough sets [14]. Pawlak's rough sets [15] are based on the notion of approximation spaces $(W, R)$, where $R$, called the indiscernibility relation, is an equivalence relation on the domain $W$ of discourse. The premise is that due to lack of complete information about the objects in the domain, it is likely that many of the objects are indistinguishable from each other. This is patent in information systems $I:=\left(W, A t, V_{A t}, f\right)$, where $A t$ is a set of attributes, $V_{A t}$ a set of values for the attributes in $A t$, and $f: W \times A t \rightarrow V_{A t}$ a function assigning values for attributes to objects of the domain. $I$ then induces an indiscernibility relation $R$ corresponding to every subset $B \subseteq A t$ :

$$
x R y \text {, if and only if } f(x, b)=f(y, b) \text {, for all } b \in B .
$$

The lower and upper approximations of a subset $X$ of the domain with respect to $R$ are defined as

$$
\underline{X}:=\{x \in W: R(x) \subseteq X\} ; \bar{X}:=\{x \in W: R(x) \cap X \neq \emptyset\} .
$$

Sets with identical approximations are said to be roughly equal, and for any $X$, the collection of all subsets of the domain roughly equal to $X$ is termed a rough set by Pawlak in [15]. 
Any logic of rough sets thus has an essential modal nature, the necessity and possibility operators capturing the lower and upper approximations in the rough set semantics respectively. In fact, for any S5 Kripke model $M=(W, e, R)$, one may observe that $e(\square \varphi)=e(\varphi)$ and $e(\diamond \varphi)=\overline{e(\varphi)}$, where $e(\varphi):=\{w \in W$ : $e(w, \varphi)=1\}$. However, unlike MEL or $\mathrm{MEL}^{+}$, rough set logics make use of the full modal language, that is, nested modalities are allowed. For instance one would use a formula such as $\diamond(p \wedge \square q)$ to refer to a set $\overline{(X \cap \underline{Y})}$.

This approach can easily be extended to rough set models based on a relation that is not necessarily an equivalence one $[17,18]$. These logics remain modal, and use nested modalities. Indeed, it is well known in modal logic [4] that, once fixed the basic axioms (PL) and (K), then each additional modal axioms corresponds a different property of the accessibility relation.

\subsection{Comparison to the Logic of Minimal Belief and Negation as Failure (MBNF)}

In [12] Lifschitz defines a simplified version of Lin and Shoham logic [13] of minimal knowledge and justified assumptions. His (nonmonotonic) logic, in the propositional version, contains two modal operators, one for minimal belief $B$ and another for negation as failure not. For positive formulas, i.e. formulas not containing not, the (monotonic) semantics is very similar to that of $\mathrm{MEL}^{+}$: semantics are given by structures $(I, S)$, where $I$ is an interpretation of propositional variables (or equivalently a set of atoms) and $S$ a set of interpretations. The author writes that, "intuitively, $I$ represents 'the real world', and $S$ the set of 'possible worlds'". As in $\mathrm{MEL}^{+}$, for a nonmodal formula $\varphi$, a structure $(I, S)$ satisfies the formula $B \varphi$ whenever each $I^{\prime} \in S$ satisfies $\varphi$. Therefore MBNF structures $(I, S)$ exactly correspond to pointed epistemic $\mathrm{MEL}^{+}$models.

The nonmonotonic semantics of MBNF is defined as to capture the notion of minimal belief. A model of a theory $T$ (set of positive formulas) is a structure $(I, S)$ such that it makes true all the formulas of $T$ and it is maximal in the sense that there is no other structure $\left(I^{\prime}, S^{\prime}\right)$ making the formulas of $T$ true and such that $S \subsetneq S^{\prime}$. For instance, the only model of $B \varphi$ in this semantics is $(I, \operatorname{Mod}(\varphi))$, while the models of $B \varphi \vee B \psi$ are $(I, \operatorname{Mod}(\varphi))$ and $(I, \operatorname{Mod}(\psi))$. Then the corresponding notion of (nonmonotonic) consequence relation is defined accordingly. For instance one has $B p \models \neg B q$ but $\{B p, B q\} \not \models \neg B q$.

Actually, MBNF models for formulas of the language of MEL (resp. MEL ${ }^{+}$) correspond to the minimum specific epistemic models in MEL (reps. pointed epistemic models in $\mathrm{MEL}^{+}$) in the sense of possibilistic logic.

\section{Conclusion}

In this paper, we argue that the usual semantics of epistemic logics based on accessibility relations is not very natural when the purpose is to reason about beliefs or incomplete knowledge entertained by an external agent, and introspection is ruled out. To this end, we have shown that the fragment $\mathrm{MEL}^{+}$(resp. 
$\mathrm{MEL}^{++}$) of the KD45 (resp. S5) logic, the richest of doxastic (resp. epistemic) logics, involving modal formulas of depth 0 or 1 can have simplified semantics that are more intuitive than equivalence relations, while the latter make sense for capturing rough sets. The connection between $\mathrm{MEL}^{+}$and MBNF clearly suggests the former has more to do with logic programming than to the mainstream modal logic tradition, as already noticed with generalized possibilistic logic (a multimodal extension of MEL), that can encode answer-set programming [5].

Acknowledgments. Godo acknowledges partial support by the Spanish MINECO project EdeTRI TIN2012-39348-C02-01.

\section{References}

1. Banerjee, M., Dubois, D.: A Simple Modal Logic for Reasoning about Revealed Beliefs. In: Sossai, C., Chemello, G. (eds.) ECSQARU 2009. LNCS, vol. 5590, pp. 805-816. Springer, Heidelberg (2009)

2. Banerjee, M., Dubois, D.: A simple logic for reasoning about incomplete knowledge. Int. J. Approximate Reasoning 55(2), 639-653 (2014)

3. Boutilier, C.: Modal logics for qualitative possibility theory. Int. J. Approximate Reasoning 10, 173-201 (1994)

4. Chellas, B.F.: Modal Logic: An Introduction. Cambridge University Press (1980)

5. Dubois, D., Prade, H., Schockaert, S.: Stable Models in Generalized Possibilistic Logic. In: Brewka, G., et al. (eds.) Proc. of KR 2012, pp. 519-529. AAAI Press (2012)

6. Fagin, R., Vardi, M.Y.: An internal semantics for modal logic. In: Proc. of STOC 1985, pp. 305-315. ACM, New York (1985)

7. Halpern, J.Y., Fagin, R., Moses, Y., Vardi, M.Y.: Reasoning About Knowledge. MIT Press (2003) (Revised paperback edition)

8. Fariñas del Cerro, L., Herzig, A.: A modal analysis of possibility theory. In: Jorrand, P., Kelemen, J. (eds.) FAIR 1991. LNCS, vol. 535, pp. 11-18. Springer, Heidelberg (1991)

9. Hájek, P.: A qualitative fuzzy possibilistic logic. Int. J. of Approximate Reasoning 12, 1-19 (1994)

10. Hájek, P., Harmancová, D., Esteva, F., Garcia, P., Godo, L.: On modal logics for qualitative possibility in a fuzzy setting. In: Lopez de Mántaras, R., Poole, D. (eds.) Proc. of UAI-94, pp. 278-285. Morgan Kaufmann, San Francisco (1994)

11. Lang, J., Marquis, P.: A forgetting-based approach. Artificial Intelligence 174, 799-823 (2010)

12. Lifschitz, V.: Minimal belief and negation as failure. Artificial Intelligence 70, 53-72 (1994)

13. Lin, F., Shoham, Y.: A logic of knowledge and justified assumptions. Artificial Intelligence 57, 271-289 (1992)

14. Orlowska, E.: A logic of indiscernibility relations. In: Skowron, A. (ed.) SCT 1984. LNCS, vol. 208, pp. 177-186. Springer, Heidelberg (1985)

15. Pawlak, Z., Skowron, A.: Rudiments of rough sets. Inf. Sciences 177, 3-27 (2007)

16. Petruszczak, A.: Simplified Kripke style semantics for modal logics K5, KB5 and KD45. Bulletin of the Section of Logic 38, 163-171 (2009)

17. Yao, Y., Lin, T.: Generalization of rough sets using modal logic. Automation and Soft Computing 2(2), 103-120 (1996)

18. Yao, Y., Wang, S., Lin, T.: A review of rough set models. In: Polkowski, L., Skowron, A. (eds.) Rough Sets and Data Mining: Analysis for Imprecise Data, pp. 47-75. Kluwer Academic Publishers (1997) 